OPEN ACCESS

Edited by:

Tim Anderson,

University of Otago, Christchurch,

New Zealand

Reviewed by:

Zhong Pei,

Sun Yat-sen University, China

Pedro Chana,

Universidad de Santiago de Chile

Chile

*Correspondence:

Huifang Shang

hfshang2002@126.com

Hai Xiong

xhxysq@126.com

Specialty section

This article was submitted to

Movement Disorders,

a section of the journal

Frontiers in Neurology

Received: 02 July 2018 Accepted: 08 October 2018

Published: 12 November 2018

Citation:

Luo X, Ou R, Dutta R, Tian Y, Xiong H and Shang $H$ (2018) Association

Between Serum Vitamin D Levels and

Parkinson's Disease: A Systematic

Review and Meta-Analysis.

Front. Neurol. 9:909.

doi: 10.3389/fneur.2018.00909

\section{Association Between Serum Vitamin D Levels and Parkinson's Disease: A Systematic Review and Meta-Analysis}

\author{
Xiaoyue Luo ${ }^{1}$, Ruwei Ou ${ }^{1}$, Rajib Dutta ${ }^{1}$, Yuan Tian ${ }^{1}$, Hai Xiong ${ }^{2 *}$ and Huifang Shang ${ }^{1 *}$ \\ ${ }^{1}$ Department of Neurology, West China Hospital, Sichuan University, Chengdu, China, ${ }^{2}$ Department of Geriatrics, The Fourth \\ Affiliated Hospital of Sichuan University, Chengdu, China
}

Background: Vitamin D is an important secosteroid which is involved the development and regulation of brain activity. Several studies have focused on exploring the relationship between serum vitamin D levels and Parkinson's disease (PD), but the conclusion remains ambiguous.

Methods: We searched observational studies that explored the association between serum vitamin D levels and PD based on PubMed, EMBASE and Cochrane library from inception through to January 2018. The quality of included studies was evaluated by using Newcastle-Ottawa Scale (NOS). Statistical analysis of this meta-analysis was performed by Stata version 12.0 and $\mathrm{R}$ software.

Results: Twenty studies with a total of 2,866 PD patients and 2,734 controls were included. Compared with controls, PD patients had lower serum vitamin D levels (WMD -3.96, 95\% Cl -5.00, -2.92), especially in higher latitude regions (WMD -4.20 , $95 \% \mathrm{Cl}-5.66,-2.75)$. Assay methods contributed significantly to high heterogeneity. Furthermore, PD patients with deficient vitamin D levels had advanced risk (OR 2.08, $95 \% \mathrm{Cl} 1.35,3.19)$ than those patients with insufficient ones $(\mathrm{OR}=1.73,95 \% \mathrm{Cl} 1.48$, 2.03). In addition, serum vitamin $D$ levels were also related to the severity of PD (WMD $-5.27,95 \% \mathrm{Cl}-8.14,-2.39)$ and the summary correlation coefficient showed strongly negative correlation $(r=-0.55,95 \% \mathrm{Cl}-0.73,-0.29)$. Moreover, the pooled correlation coefficient revealed that serum vitamin D levels were also negatively correlated to the Unified Parkinson's Disease Rating Scale III (UPDRS III) ( $r=-0.36,95 \% \mathrm{Cl}-0.53$, $-0.16)$, but did not correlate with the duration of PD $(P=0.37)$ and age of patients $(P=0.49)$.

Conclusion: Serum vitamin D levels are inversely associated with the risk and severity of PD. Our results provided an updated evidence of association between low vitamin $\mathrm{D}$ levels and PD and prompt the adjunctive therapeutic decisions about vitamin $\mathrm{D}$ replacement in PD.

Keywords: Parkinson's disease, vitamin D, meta-analysis, observational studies, motor symptom 


\section{INTRODUCTION}

Parkinson's disease (PD) is a common complex neurodegenerative disorder characterized by a variety of motor and non-motor symptoms. As the disease progresses, PD symptoms can significantly affect the quality of life. Recently, it was reported that the median age-standardized annual incident rate of PD has raised to 14 per 100,000 people in high-income countries (1), indicating that the burden of developing PD within a family is on the rise. To date, the exact pathogenesis of PD is still unclear, and no curative treatment is available. Thus, slowing down the progression of PD is of utmost importance.

Vitamin D is a group of steroid derivatives, which can regulate the metabolism of calcium and phosphate. Recently, there are increasing evidences which show that Vitamin D plays an important role in cell modulation, such as proliferation, differentiation, immunoregulation and so on (2). In addition, an animal study found that vitamin $\mathrm{D}$ might have a neuroprotective function (3). Vitamin D deficiency is common in PD (4). However, it is argued whether vitamin $\mathrm{D}$ insufficiency is related to the etiology of PD. Two recent longitudinal studies suggested that the reduced serum vitamin D levels are significantly associated with risk of developing $\operatorname{PD}(5,6)$. This evidence provides the possibility of evaluating the risk of $\mathrm{PD}$ by using serum vitamin $\mathrm{D}$ levels as a biomarker.

Although three previous meta-analysis have focused on the relationship between serum vitamin D levels and PD (7-9), it did not include enough studies, extract all related data, and analyze the association between possible confounding factor and the indicator of PD progression. In our study, we therefore enlarged the number of studies and conducted a deeper systematic review and meta-analysis to assess the association between the serum vitamin $\mathrm{D}$ levels and $\mathrm{PD}$, not only the susceptibility but also the severity of PD.

\section{METHODS}

\section{Search Strategy}

We searched the major biomedical databases, including PubMed, EMBASE, and Cochrane library for all related studies published before January 2018. The keywords used for searching were "Parkinson's disease" or "PD" combined with "vitamin D," "vit D," "cholecalciferol," "ergocalciferols" "hydroxycholecalciferol" or "ergocalciferol." To avoid missing literatures, we also looked through the references of relevant articles. Two researchers (Xiaoyue Luo and Yuan Tian) filtered the articles independently to identify qualified articles. If any disagreement or conflict, the third researcher (Ruwei $\mathrm{Ou}$ ) decided whether the article was included in this meta-analysis. Our meta-analysis was registered with PROSPERO (registration number CRD42018086043).

\section{Definitions}

All recruited patients met the diagnosis criteria of PD, we used the Unified Parkinson's Disease Rating Scale III(UPDRS III) to assess the motor symptoms. The severity of PD was defined on the basis of the Hoehn \& Yahr (H\&Y) scale and scores

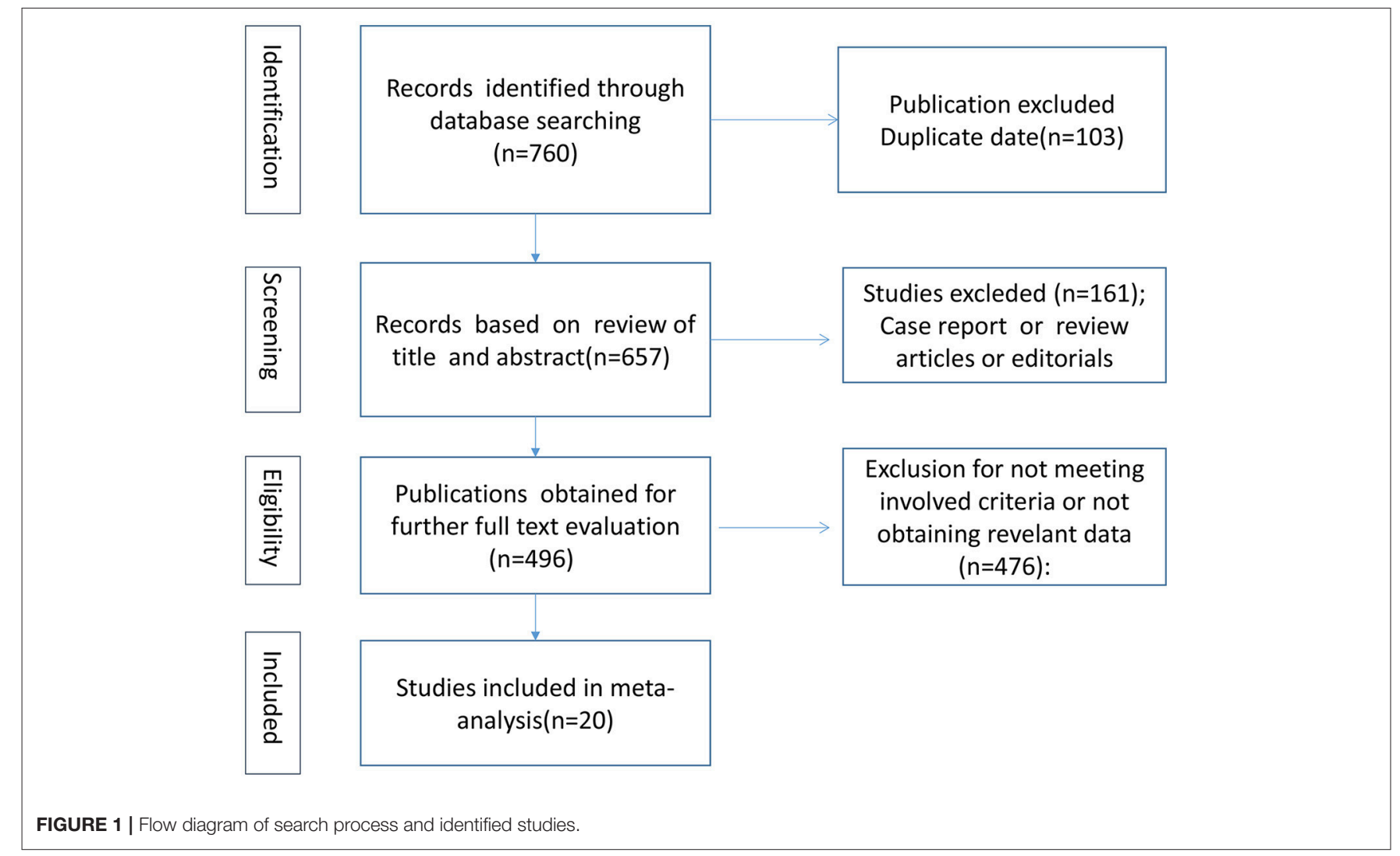


$>3$ indicated severe stages of PD (10). The controls were agematched healthy individuals. Serum 25-hydroxyvitamin D was taken as a indicator of vitamin D status worldwide (11). Serum $25(\mathrm{OH}) \mathrm{D}$ concentration $<20 \mathrm{ng} / \mathrm{ml}(50 \mathrm{nmol} / \mathrm{l})$ was regarded as vitamin D deficiency, between $20 \mathrm{ng} / \mathrm{ml} \mathrm{(50} \mathrm{nmol} / \mathrm{l})$ and $30 \mathrm{ng} / \mathrm{ml}$ ( $75 \mathrm{nmol} / \mathrm{l})$ was considered as vitamin D insufficiency, and above $30 \mathrm{ng} / \mathrm{ml}(75 \mathrm{nmol} / \mathrm{l})$ was considered as the normal level (11). The unit of $\mathrm{nmol} / \mathrm{l}$ was converted to the value of $\mathrm{ng} / \mathrm{ml}$ in our meta-analysis.

\section{Selection and Exclusion Criteria}

We finally included 20 articles showed in Table S1 in Supplementary Material by scanning the titles, abstracts and further reviewing the full texts. The flow chart of selection process is presented in Figure 1. All of the included articles met the following criteria: (1) cohort, case-control, or cross-sectional study; (2) to investigate the association between serum $25(\mathrm{OH}) \mathrm{D}$ levels and PD; (3) higher quality papers; and (4) published in English. The exclusion criteria were listed as follows: (1) case reports, reviews or editorial; (2) cells or animal studies; (3) the serum levels of vitamin $\mathrm{D}$ calculated by other indicators and not by $25(\mathrm{OH}) \mathrm{D}$; and (4) incomplete data.

\section{Data Extraction}

The following data was extracted including first author, publication year, study design, sample size, methods of test, latitude, H\&Y stages, UPDRS III scores, serum vitamin D levels and so on. To reduce mistakes and bias, two authors extracted data separately and identified by a third researcher. If the articles did not provide enough data of our interest, we tried our best to contact the author.

\section{Quality Evaluation}

We used Newcastle-Ottawa Scale (NOS) (12) to assess the quality of evidence. It contains three parts which includes selection (0-4 stars), comparability (0-2 stars) and exposure/outcome (0-3 stars). The NOS scores of $1-3,4-6$, and 7-9 indicated low, medium and high quality of studies, respectively (13). As displayed in Table S2 in Supplementary Material, this metaanalysis consisted of articles with stars $\geq 6$ and could be considered as higher methodological quality. Any dissension among researchers was resolved by final discussion to reach a consensus.

\section{Statistical Analyses}

For dichotomous data, the odds ratio (OR) and 95\% CI were pooled. For continuous data, the mean difference (MD) or the standardized mean difference (SMD) and 95\% CI were calculated. In addition, we also combined correlation coefficient (r) using inverse variance method as reported before (14). Heterogeneity was evaluated by $\mathrm{Chi}^{2}$ test and $\mathrm{I}^{2}$ squared. $I^{2}>50 \%$ or $P<0.1$ represented substantial heterogeneity and random effects model was choosen under this circumstance. Otherwise, fixed effects model was utilized. If high heterogeneity existed, subgroup and meta regression analysis was carried out. Sensitivity analysis was also done by removing the study one by one, switching effects model and exchanging of statistical values.
The publication bias was estimated by Egger's test and funnel's plot if included papers $\geq 10$ according to Cochrane handbook. All statistical analysis of this meta-analysis was performed by Stata version 12.0 (StataCorp LP, College Station, TX, USA) and R software (https://www.r-project.org/).

\section{RESULTS}

\section{Search Results and Study Characteristics}

A total of 760 articles were selected according to the search strategy mentioned before (Figure 1). After we reviewed the titles, abstracts and full texts, 20 studies (15-34) including 2,866 PD patients and 2,734 controls met our inclusion criteria. Fifteen articles $(15,17-19,21,23-26,28,29,31-34)$ provided serum 25( $\mathrm{OH}) \mathrm{D}$ levels of PD patients compared with those of controls. Seven included articles $(17,18,21,29,31-33)$ analyzed vitamin D insufficiency between PD patients and controls, while 10 articles $(17-21,28,29,31,33,34)$ assessed vitamin D deficiency in PD patients compared with controls. Six included papers $(16,17,19$, $25,26,30)$ evaluated the association between serum vitamin $\mathrm{D}$ levels and the severity of PD. At last, 6 essays (19, 22, 25-28) were identified for exploring the correlative factors of vitamin D, including H\&Y stages, UPDRS III scores, age of patients and disease duration.

\section{Serum Vitamin D Levels in PD Patients}

Fifteen studies $(15,17-19,21,23-26,28,29,31-34)$ provided data of serum vitamin D levels between PD patients $(n=2,436)$ and age matched controls $(n=2,567)$. We only included the paper with the most complete description of data (23) since four papers had the same first author's name (Sato Y) (2326). Our results showed that the serum vitamin D levels in PD patients were significantly lower than those in controls (WMD $-5.64,95 \% \mathrm{CI}-8.65,-2.62)$, which had marked heterogeneity $\left(I^{2}=98.6 \%\right)$. Further meta funnel research found the funnel plot was asymmetric and the study of Sato et al. (23) deviated much from it. After we removed this study, however, the result did not change (WMD $-3.96,95 \% \mathrm{CI}-5.00,-2.92, I^{2}=82.6 \%$, Figure 2). To explore the high heterogeneity, the subgroup analysis of assay methods was conducted. It could extremely explain the source of heterogeneity. We also studied the subgroup analysis of different latitude, which indicated that PD patients in higher latitude area (WMD $-4.20,95 \% \mathrm{CI}-5.66,-2.75$ ) had lower serum vitamin $\mathrm{D}$ concentrations than those in lower latitude ones (WMD $-3.45,95 \% \mathrm{CI}-5.75,-1.15$, Figure 3). Although high heterogeneity existed, the meta regression showed that the latitude degree was not the main source of heterogeneity (adjust- $\mathrm{R}^{2}=16.26 \%, P=0.636$ ). Also, publication bias was detected by Egger's test and the outcome showed no significant bias $(P=0.30)$. Sensitivity analyses revealed the pooled effects were robust, and so did the trim and fill method.

\section{Vitamin D Insufficiency and PD Risk}

Seven articles (17, 18, 21, 29, 31-33) including 1,613 PD patients and 2,026 controls evaluated the influence of vitamin $\mathrm{D}$ insufficiency on the risk of PD. Due to statistical heterogeneity $\left(I^{2}=70.9 \%\right)$, the random effects model was selected. The results 


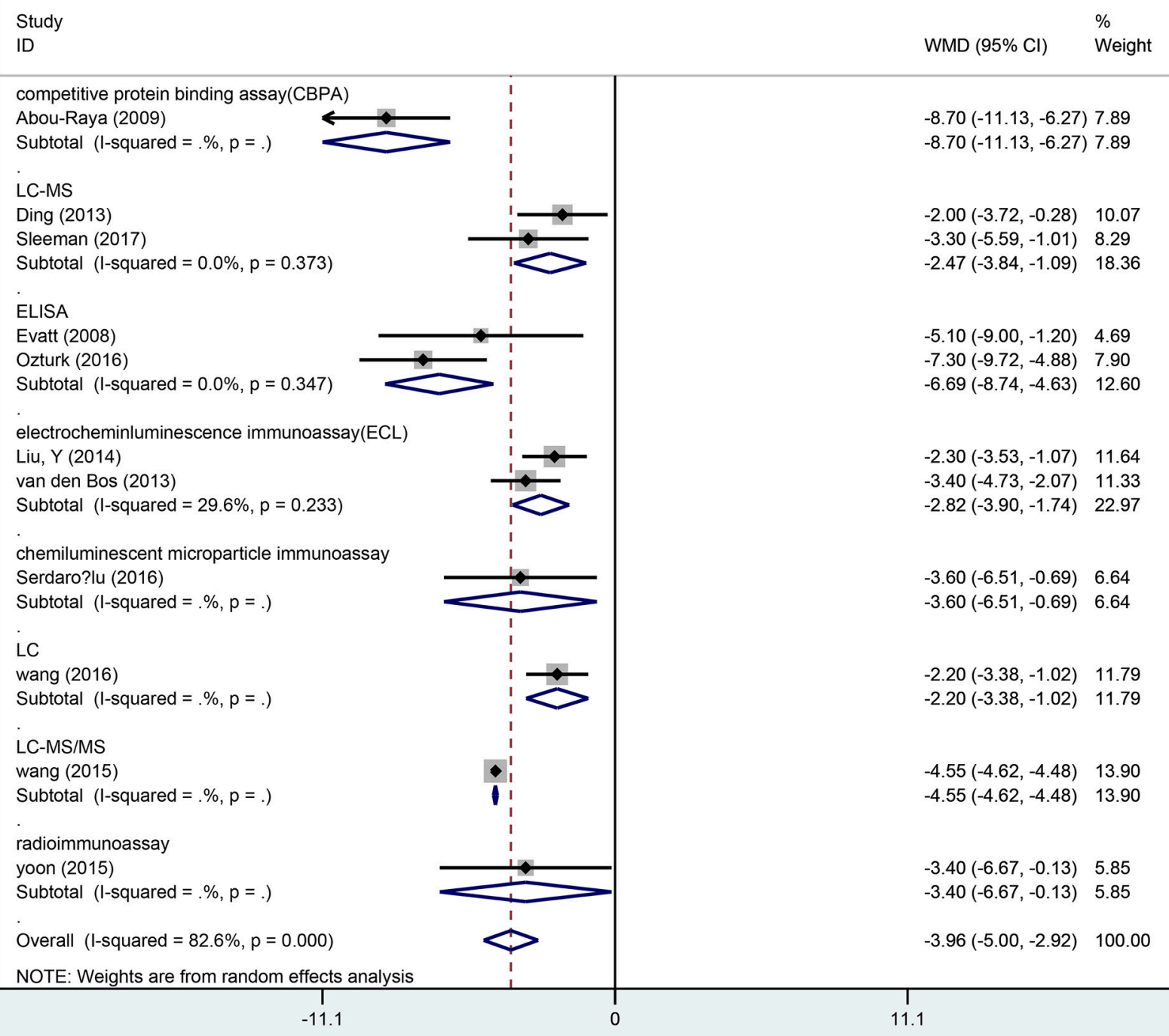

FIGURE 2 | Forest plot of vitamin D levels between PD patients and controls divided by assay methods.

revealed a pooled OR of $1.52(95 \% \mathrm{CI} 1.10,2.10, P=0.012)$, suggesting that vitamin $\mathrm{D}$ insufficiency can increase the risk of PD. Due to the limited number of articles, publication bias was not computed. The sensitivity analyses showed no excessive study could alert the final consequence. However, when we removed the study of Ozturk et al. (21), the heterogeneity $\left(I^{2}\right)$ decreased to $31 \%(\mathrm{OR}=1.73,95 \% \mathrm{CI} 1.48,2.03$, Figure 4$)$.

\section{Vitamin D Deficiency and PD Risk}

Ten articles (17-21, 28, 29, 31, 33, 34) including 1,879 PD patients and 1,978 controls reported the association between the vitamin $\mathrm{D}$ deficiency and the risk of $\mathrm{PD}$. A remarkable association was found that vitamin $\mathrm{D}$ deficient subjects had a 2 -fold increased risk of PD than controls using the random effects analysis (OR 2.08,
95\%CI 1.35, 3.19, $I^{2}=84.7 \%$, Figure 5), which was also higher than insufficient groups. In addition, Egger's test identified no publication bias $(P=0.572)$. Sensitivity analyses comfirmed the similar results.

\section{Serum Vitamin D Levels and the Severity of PD}

Six articles $(16,17,19,25,26,30)$ including 991 PD patients investigated the difference in serum vitamin $\mathrm{D}$ levels among different severity of PD groups based on H\&Y scale. We only included the paper which provided more detailed descriptions (25) since the two articles $(25,26)$ were published by the same first author. Random effect model illustrated serum vitamin D 


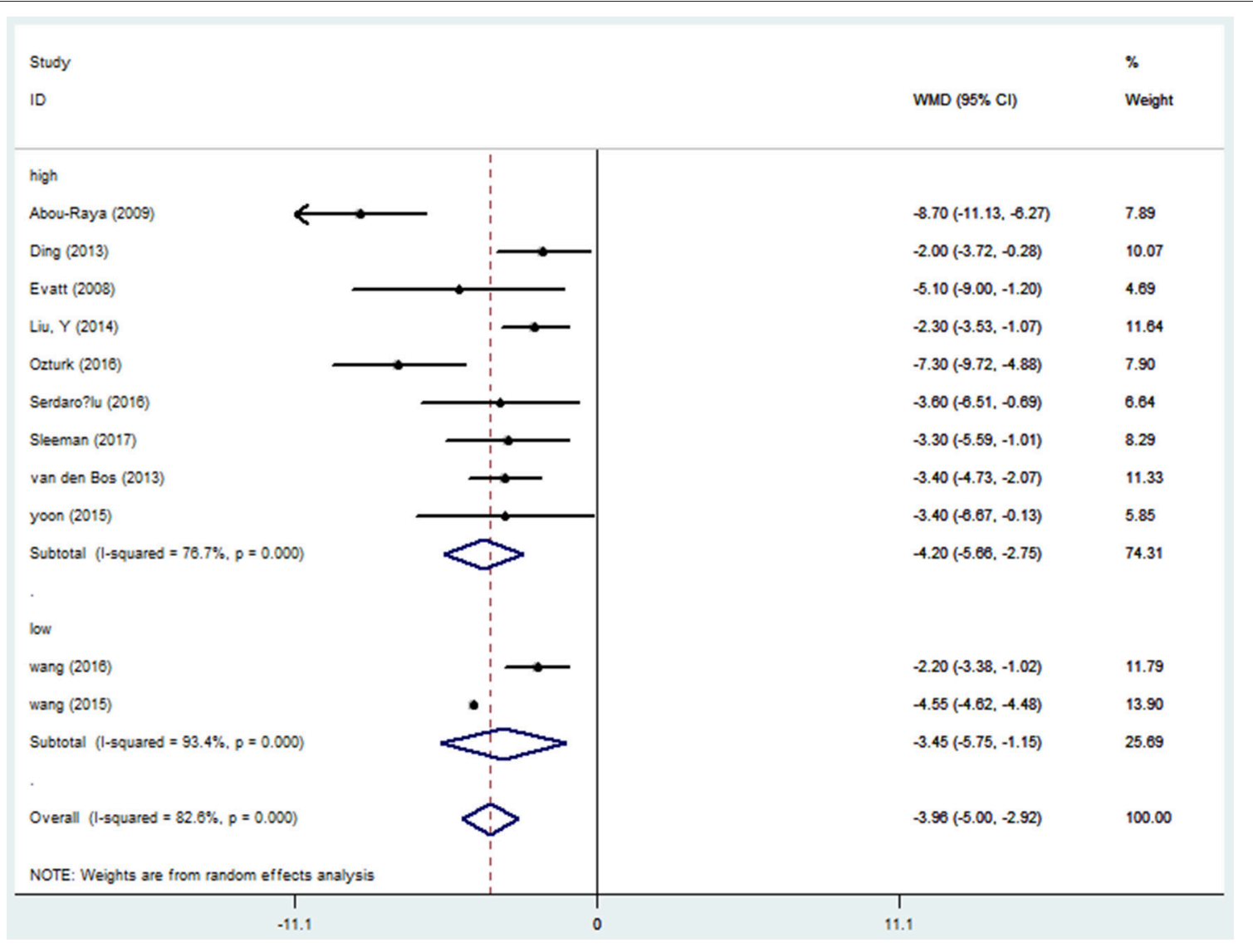

FIGURE 3 | Forest plot of vitamin D levels between PD patients and controls divided by latitude.

concentrations were significantly low in the higher $\mathrm{H} \& \mathrm{Y}$ stages group (WMD-5.27, 95\%CI $-8.14,-2.39$, Figure 6). The high degree of heterogeneity $\left(I^{2}=92.6 \%\right)$ could be greatly explained by assay methods. The publication bias and sensitivity analyses were not detected because of small number of studies.

\section{Correlative Factors of Serum Vitamin D Levels in PD}

Five articles (22, 25-28) involving 244 PD patients explored the relationship between serum vitamin $\mathrm{D}$ concentrations and H\&Y stages. Because the two articles $(25,26)$ were published by the same first author (Sato Y), we only included the more comprehensive one (25). The pooled correlation coefficient (spearman) was -0.55 but it displayed significant heterogeneity $\left(95 \% \mathrm{CI}-0.73,-0.29, I^{2}=74 \%\right.$, Figure 7$)$, so the random effect model was used. Furthermore, fix effect analysis of two studies $(22,28)$ which investigated the association of UPDRS III scores in PD patients with vitamin D levels indicated the pooled correlation coefficient (person) was -0.36 (95\%CI $-0.53,-0.16$, $I^{2}=0 \%$, Figure 8). However, serum vitamin $\mathrm{D}$ concentrations were not associated with duration of $\operatorname{PD}(19,28)(P=0.37)$ and age of patients $(28)(P=0.49)$. The sensitivity analyses showed consistent results and we could not make publication bias for finite studies.

\section{DISCUSSION}

Although three previous meta-analysis conducted the association between vitamin D status and PD (7-9), they did not analyze the relationship of vitamin $\mathrm{D}$ deficiency and the severity of PD. In addition, limited number of studies were included in the three previous meta-analysis, i.e., Lv et al. (7) recruited two studies $(17,18)$, Shen et al. (8) included three studies $(17,18,33)$, and Rimmelzwaan et al. (9) included eight studies (15, 23, 26, 27, 31, 35-37). The current meta-analysis included large number of studies and not only assessed the association between serum vitamin $\mathrm{D}$ levels and the risk of $\mathrm{PD}$ but also the association between serum vitamin $\mathrm{D}$ levels and the severity of PD.

We found that the serum 25(OH)D levels in PD patients were significant lower than those in controls, which was consistent with the previous study (7). However, there was a high heterogeneity originating from assay methods. We further found that people who lived in higher latitude regions have lower serum 25(OH)D concentrates compared with people who lived in lower latitude regions, which is in accordance with the traditional theory that lower latitude area gets more sunlight which can enhance vitamin $\mathrm{D}$ biosynthesis. With regard to lower vitamin D levels in PD patients, we should admit limited mobility has an effect on low level of vitamin D due to less sunlight 


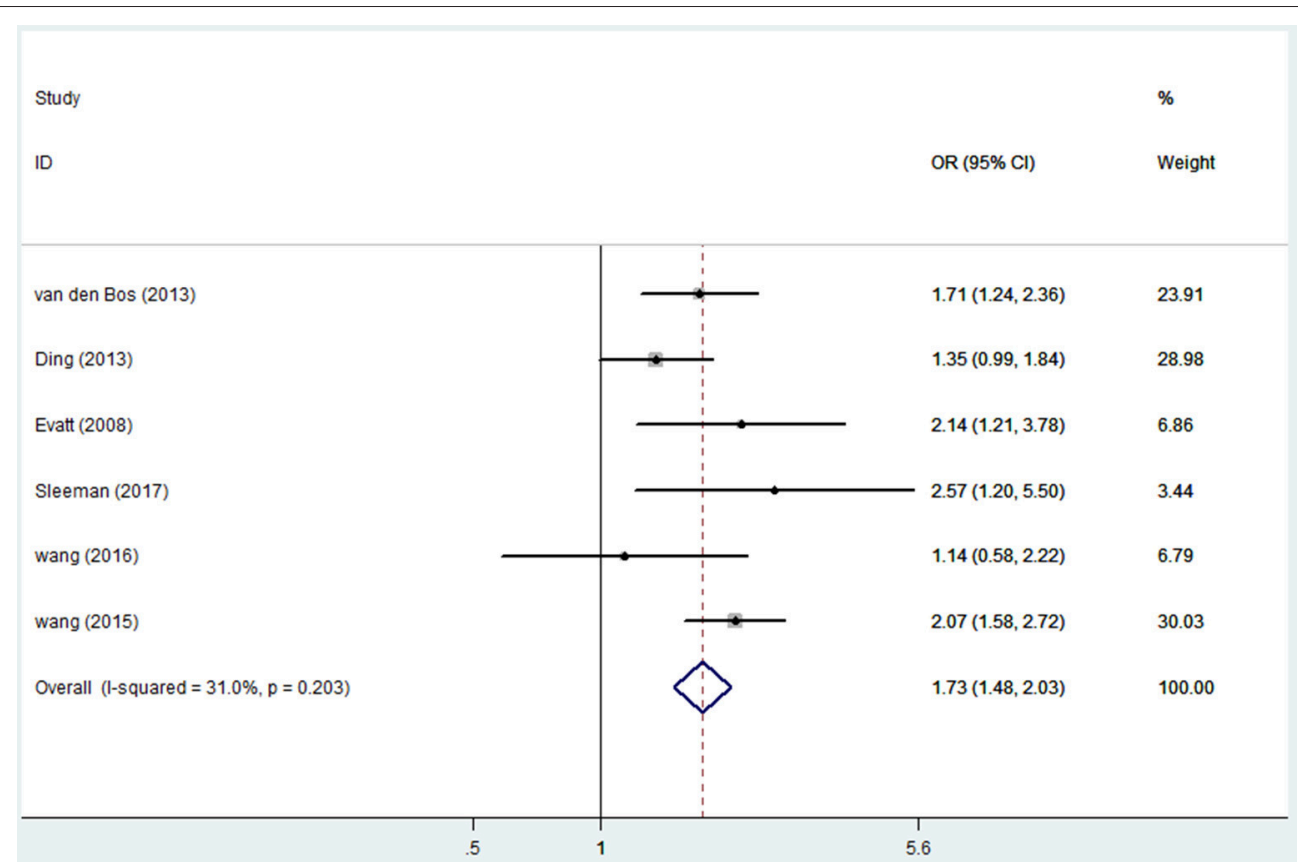

FIGURE 4 | Forest plot of insufficient vitamin D levels and risk of PD.

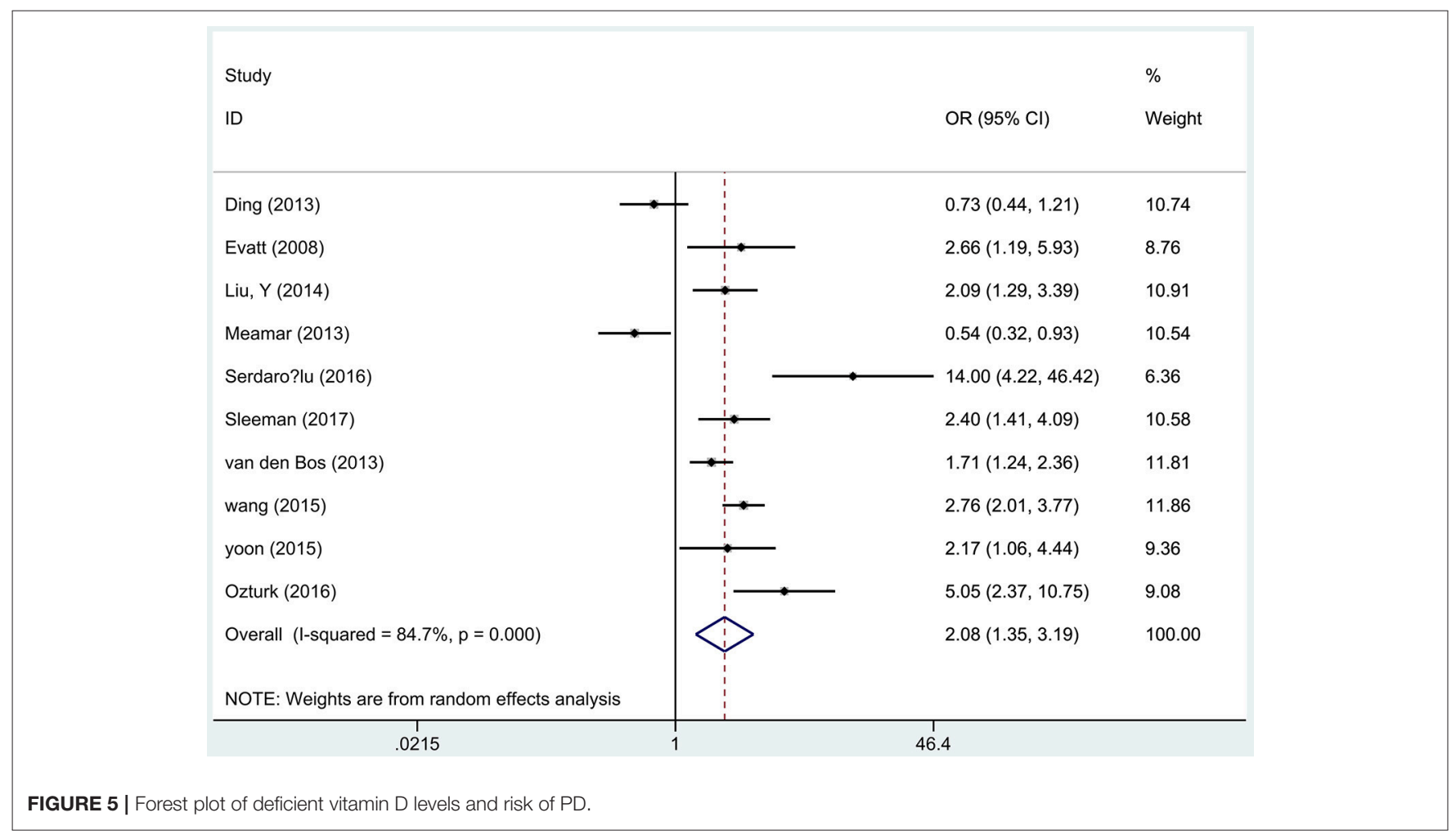

exposure in PD patients. However, high prevalence of deficient vitamin $\mathrm{D}$ concentrations also exist in non-disabling and early PD subjects even if they had normal ambulation (36). Wang et al. (33) reported serum vitamin D2 levels obtained mainly from diet rather than sunlight. Gastrointestinal dysfunction including dysphagia, delayed gastric emptying and constipation could result in poor digestion and absorption, which may also contribute to the low serum vitamin D levels in PD patients (38). 


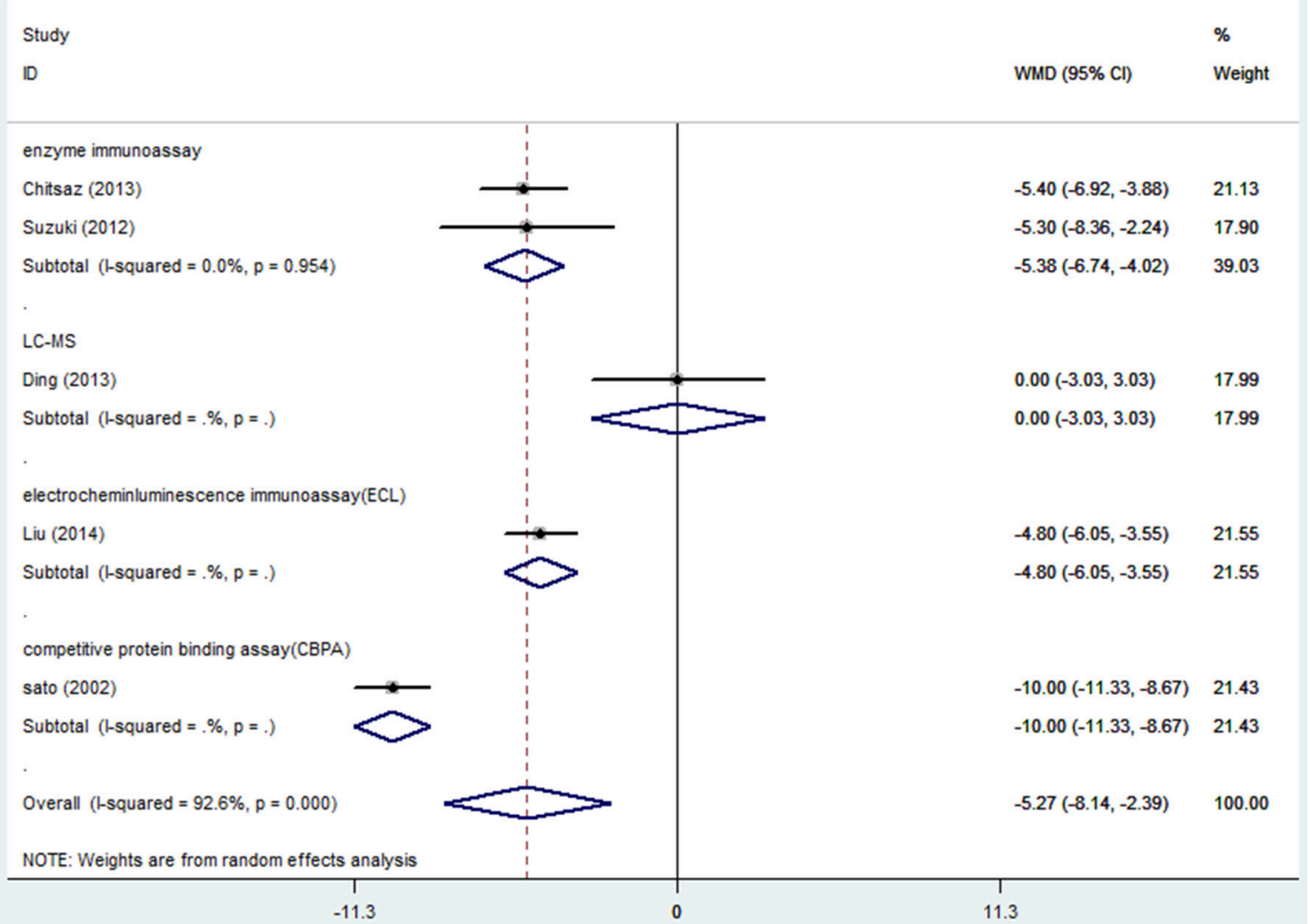

FIGURE 6 | Forest plot of vitamin D levels in PD patients with different severity.

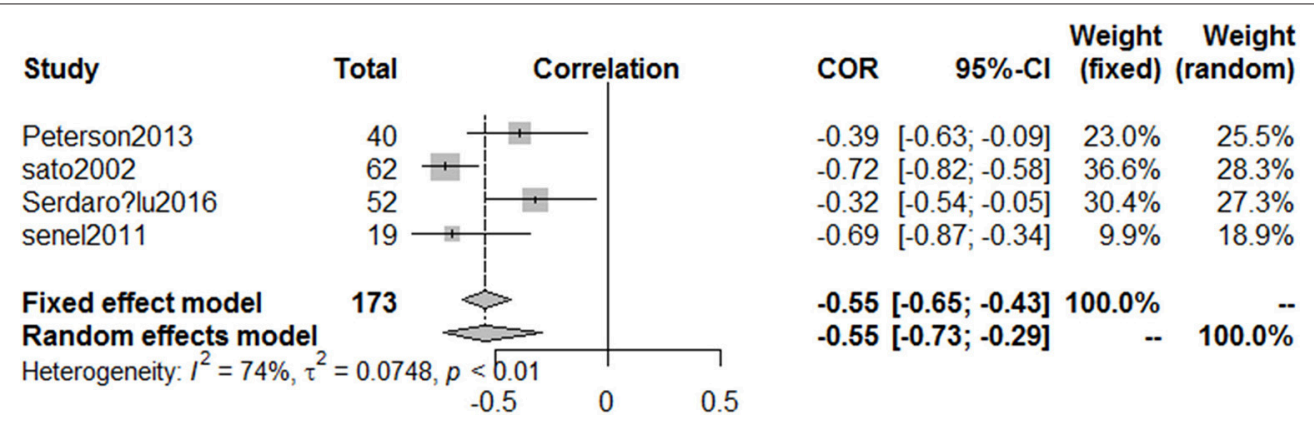

FIGURE 7 | Forest plot of the correlation coefficient between vitamin D levels and different HYstages in PD patients.

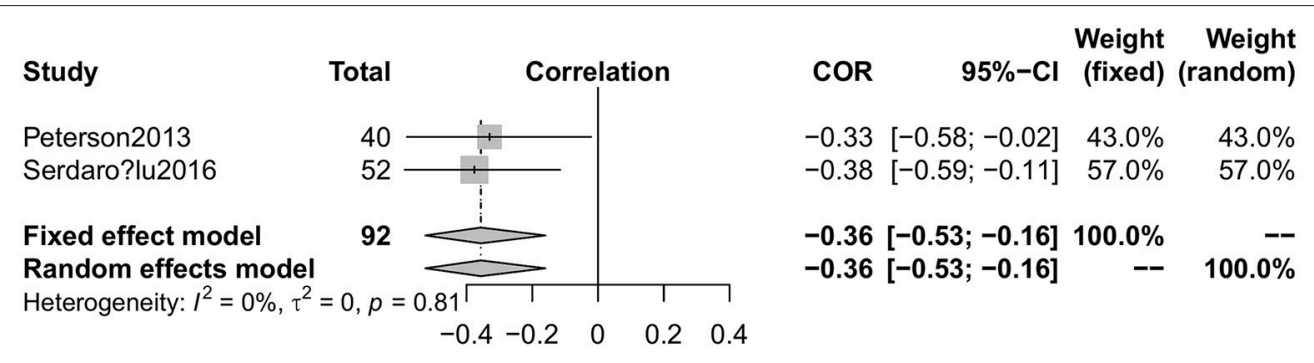

FIGURE 8 | Forest plot of the correlation coefficient between vitamin D levels and mUPDRS scores in PD patients. 
In addition, malnutrition, failure of atrophic skin to produce vitamin $\mathrm{D}$ as well as comorbidities, such as renal or hepatic dysfunction in the elderly could also influence the vitamin D concentrations (39-41).

Both vitamin $\mathrm{D}$ insufficiency and deficiency can increase the risk of $\mathrm{PD}$, however there is an increased risk of $\mathrm{PD}$ in vitamin $\mathrm{D}$ deficiency as compared to insufficiency vitamin $\mathrm{D}$. The serum 25(OH)D levels were remarkably distinct among different severity PD groups. And they also were negatively correlated with H\&Y stages and UPDRS III scores, but not the disease duration and the age of PD patients. Furthermore, the possible biological mechanisms for explaining vitamin D status contributing to the severity of PD are complicated.

First, it is of utmost importance to understand that only active vitamin $\mathrm{D}$ is responsible for all the normal physiological function inside our body. And the active process of vitamin D is regulated by $1 \alpha$-hydroxylase in kidney, an enzyme which can change $25(\mathrm{OH}) \mathrm{D}$ into $1,25(\mathrm{OH}) 2 \mathrm{D} 3$. As for vitamin $\mathrm{D}$ receptor (VDR) mediated by special VDR genes, VDRs knockout mice showed significantly impaired motor functions (42). Both $1 \alpha-$ hydroxylase and VDR are expressed in human brain especially in the substantia nigra and hypothalamus (43). An animal study indicated that vitamin $\mathrm{D}$ increased tyrosine hydroxylase positive cells and inhibited inflammatory responses to ameliorate PD symptoms (44). Furthermore, single nucleotide polymorphisms (SNPs) in the VDR gene also have an effect on the serum vitamin D levels in PD patients $(45,46)$. Second, vitamin D plays an important role in neuroprotective actions through promoting the release of glial cell-derived neurotrophic factor (GDNF) and other trophic factors. Besides, vitamin $\mathrm{D}$ can indirectly restore dopaminergic circuits by increasing these neurotrophic factors. As a group of fat-soluble secosteroids, it is also easy to cross the blood brain barrier and exert its physiological effects (47). Third, lewy bodies are abnormal aggregates of alpha synuclein that develops in neurons in PD. Vitamin D analogs can decrease intracellular-free Ca (II) and downregulate the expression of calbindin-D28k to reduce $\alpha$-synuclein aggregation (48). However, so far its pathogenesis has not been completely figured out.

There are some limitations in our meta-analysis that should be considered. Assay methods contributed largely to the discrepancy among these studies. There are many ways to detect the serum vitamin D levels which have different sensitivities and specificities (49). Till now there is no single ideal technique to synthesize

\section{REFERENCES}

1. Ascherio A, Schwarzschild MA. The epidemiology of Parkinson's disease: risk factors and prevention. Lancet Neurol. (2016) 15:1257-72. doi: 10.1016/s1474-4422(16)30230-7

2. Samuel S, Sitrin MD. Vitamin D's role in cell proliferation and differentiation. Nutr Rev. (2008) 66:S116-24. doi: 10.1111/j.1753-4887.2008.00094.x

3. Calvello R, Cianciulli A, Nicolardi G, De Nuccio F, Giannotti L, Salvatore R, et al. Vitamin D treatment attenuates neuroinflammation and dopaminergic neurodegeneration in an animal model of Parkinson's disease, shifting M1 to M2 microglia responses. J Neuroimmune Pharmacol. (2017) 12:327-39. doi: $10.1007 /$ s11481-016-9720-7 the strengths of different test methods. Further attention should be paid in this area so that we can better explore the issue of vitamin $\mathrm{D}$ in $\mathrm{PD}$. As for other confounding factors (e.g., sex, race, Body Mass Index, season, education, anti-PD drugs), we could not conclude anything else due to incomplete data. We only summarized the information from H\&Y stages and UPDRS III scores, other indexes which were identically vital to assess PD symptoms, such as total UPDRS, UPDRS II, non-motor symptoms scale and so on. Relative studies are rare so that we could not do further analysis. We have to cautiously explain the finding of the relationship between vitamin D levels and duration of PD and age of PD patients. More studies are needed to verify our conclusion due to the small sample size in the meta-analysis. In addition, we did not analyze the association of PD with vitamin D from different sources i.e., vitamin D2 (dietary) and vitamin D3 (mainly sunlight). Finally, there are only a few studies which focused on the vitamin D supplement and its relation to PD (5052 ), and only one double blinded RCT study (50). Our current meta-analysis cannot elucidate the role of vitamin replacement.

\section{CONCLUSION}

In summary, our meta-analysis indicated that low serum vitamin D levels were directly correlated to the severity of symptoms of PD and increased risk of developing PD. Our results provide an incremental evidence to evaluate the role of vitamin $\mathrm{D}$ in the progression of $\mathrm{PD}$. As a potential biomarker for $\mathrm{PD}$, it should be considered to monitor the serum vitamin $\mathrm{D}$ levels. However, more high quality longitudinal randomized researches are urgently needed to address the necessary of vitamin D replacement in $\mathrm{PD}$.

\section{AUTHOR CONTRIBUTIONS}

$\mathrm{XL}, \mathrm{YT}$, and RO did the literature review. XL did the statistical analysis. All authors have significantly contributed discussions on the manuscript and reviewed the paper during the writing process.

\section{SUPPLEMENTARY MATERIAL}

The Supplementary Material for this article can be found online at: https://www.frontiersin.org/articles/10.3389/fneur. 2018.00909/full\#supplementary-material et al. Vitamin D and ageing: neurological issues. Neuropsychobiology (2010) 62:139-50. doi: 10.1159/000318570

5. Knekt P, Kilkkinen A, Rissanen H, Marniemi J, Saaksjarvi K, Heliovaara M. Serum vitamin D and the risk of Parkinson disease. Arch Neurol. (2010) 67:808-11. doi: 10.1001/archneurol.2010.120

6. Shrestha S, Lutsey PL, Alonso A, Huang X, Mosley TH Jr., Chen H. Serum 25hydroxyvitamin D concentrations in mid-adulthood and Parkinson's disease risk. Mov Disord. (2016) 31:972-8. doi: 10.1002/mds.26573

7. Lv Z, Qi H, Wang L, Fan X, Han F, Wang H, et al. Vitamin D status and Parkinson's disease: a systematic review and meta-analysis. Neurol Sci. (2014) 35:1723-30. doi: 10.1007/s10072-014-1821-6 
8. Shen L, Ji HF. Associations between Vitamin D status, supplementation, outdoor work and risk of Parkinson's disease: a meta-analysis assessment. Nutrients (2015) 7:4817-27. doi: 10.3390/nu7064817

9. Rimmelzwaan LM, van Schoor NM, Lips P, Berendse HW, Eekhoff EM. Systematic review of the relationship between vitamin D and Parkinson's disease. J Parkinsons Dis. (2016) 6:29-37. doi: 10.3233/jpd-150615

10. Martinez-Martin P, Rodriguez-Blazquez C, Mario A, Arakaki T, Arillo VC, Chana P, et al. Parkinson's disease severity levels and MDS-Unified Parkinson's Disease Rating Scale. Parkinsonism Relat Disord. (2015) 21:50-4. doi: 10.1016/j.parkreldis.2014.10.026

11. Holick MF, Binkley NC, Bischoff-Ferrari HA, Gordon CM, Hanley DA, Heaney RP, et al. Evaluation, treatment, and prevention of vitamin D deficiency: an Endocrine Society clinical practice guideline. J Clin Endocrinol Metab. (2011) 96:1911-30. doi: 10.1210/jc.2011-0385

12. Wells GA, Shea B, O'Connell D, Peterson J, Welch V, Losos M, et al. The Newcastle-Ottawa Scale (NOS) for Assessing the Quality of Nonrandomised Studies in Meta-analyses (2014). Available online at: http://www.ohri.ca/ programs/clinical_epidemiology/oxford.asp

13. Viale L, Allotey J, Cheong-See F, Arroyo-Manzano D, McCorry D, Bagary M, et al. Epilepsy in pregnancy and reproductive outcomes: a systematic review and meta-analysis. Lancet (2015) 386:1845-52. doi: 10.1016/s0140-6736(15)00045-8

14. Chalkidou A, Landau DB, Odell EW, Cornelius VR, O’Doherty MJ, Marsden PK. Correlation between Ki-67 immunohistochemistry and 18Ffluorothymidine uptake in patients with cancer: a systematic review and metaanalysis. Eur J Cancer (2012) 48:3499-513. doi: 10.1016/j.ejca.2012.05.001

15. Abou-Raya S, Helmii M, Abou-Raya A. Bone and mineral metabolism in older adults with Parkinson's disease. Age Ageing (2009) 38:675-80. doi: 10.1093/ageing/afp137

16. Chitsaz A, Maracy M, Basiri K, Izadi Boroujeni M, Tanhaei AP, Rahimi M, et al. 25-hydroxyvitamin d and severity of Parkinson's disease. Int J Endocrinol. (2013) 2013:689149. doi: 10.1155/2013/689149

17. Ding H, Dhima K, Lockhart KC, Locascio JJ, Hoesing AN, Duong K, et al. Unrecognized vitamin D3 deficiency is common in Parkinson disease: Harvard Biomarker Study. Neurology (2013) 81:1531-7. doi: 10.1212/WNL.0b013e3182a95818

18. Evatt ML, Delong MR, Khazai N, Rosen A, Triche S, Tangpricha V. Prevalence of vitamin d insufficiency in patients with Parkinson disease and Alzheimer disease. Arch Neurol. (2008) 65:1348-52. doi: 10.1001/archneur.65. 10.1348

19. Liu Y, Zhang BS. Serum 25-hydroxyvitamin D predicts severity in Parkinson's disease patients. Neurol Sci. (2014) 35:67-71. doi: 10.1007/s10072-013-1539-x

20. Meamar R, Maracy M, Chitsaz A, Ghazvini MR, Izadi M, Tanhaei AP. Association between serum biochemical levels, related to bone metabolism and Parkinson's disease. J Res Med Sci. (2013) 18(Suppl. 1):S39-42.

21. Ozturk EA, Gundogdu I, Tonuk B, Kocer BG, Tombak Y, Comoglu S, et al. Bone mass and vitamin D levels in Parkinson's disease: is there any difference between genders? J Phys Ther Sci. (2016) 28:2204-9. doi: 10.1589/jpts.28.2204

22. Peterson AL, Mancini M, Horak FB. The relationship between balance control and vitamin D in Parkinson's disease-a pilot study. Mov Disord. (2013) 28:1133-7. doi: 10.1002/mds.25405

23. Sato Y, Honda Y, Iwamoto J, Kanoko T, Satoh K. Abnormal bone and calcium metabolism in immobilized Parkinson's disease patients. Mov Disord. (2005) 20:1598-603. doi: 10.1002/mds.20658

24. Sato Y, Kaji M, Tsuru T, Oizumi K. Risk factors for hip fracture among elderly patients with Parkinson's disease. J Neurol Sci. (2001) 182:89-93. doi: 10.1016/s0531-5131(03)00110-9

25. Sato Y, Kaji M, Tsuru T, Satoh K, Kondo I. Vitamin K deficiency and osteopenia in vitamin D-deficient elderly women with Parkinson's disease. Arch Phys Med Rehab. (2002) 83:86-91. doi: 10.1053/apmr.2002.27376

26. Sato Y, Kikuyama M, Oizumi K. High prevalence of vitamin D deficiency and reduced bone mass in Parkinson's disease. Neurology (1997) 49:1273-8.

27. Senel K, Alp F, Baykal T, Melikoglu M, Erdal A, Ugur M. Preliminary study: Is there a role of vitamin D in parkinson disease? Osteoporos Int. (2011) 22:S167. doi: 10.1007/s00198-011-1567-4

28. Serdarolu Beyazal M, Kirbaş S, Tüfekçi A, Devrimsel G, Küçükali Türkyilmaz A. The relationship of vitamin D with bone mineral density in Parkinson's disease patients. Eur Geriatr Med. (2016) 7:18-22. doi: 10.1016/j.eurger.2015.08.002

29. Sleeman I, Aspray T, Lawson R, Coleman S, Duncan G, Khoo TK, et al. The role of vitamin D in disease progression in early Parkinson's disease. J Parkinsons Dis. (2017) 7:669-75. doi: 10.3233/jpd-171122

30. Suzuki M, Yoshioka M, Hashimoto M, Murakami M, Kawasaki K, Noya $\mathrm{M}$, et al. 25-hydroxyvitamin D, vitamin D receptor gene polymorphisms, and severity of Parkinson's disease. Mov Disord. (2012) 27:264-71. doi: 10.1002/mds. 24016

31. van den Bos F, Speelman AD, van Nimwegen M, van der Schouw YT, Backx FJ, Bloem BR, et al. Bone mineral density and vitamin D status in Parkinson's disease patients. J Neurol. (2013) 260:754-60. doi: 10.1007/s00415-0126697-x

32. Wang J, Yang D, Yu Y, Shao G, Wang Q. Vitamin D and sunlight exposure in newly-diagnosed Parkinson's disease. Nutrients (2016) 8:142. doi: $10.3390 /$ nu8030142

33. Wang L, Evatt ML, Maldonado LG, Perry WR, Ritchie JC, Beecham GW, et al. Vitamin D from different sources is inversely associated with Parkinson disease. Mov Disord. (2015) 30:560-6. doi: 10.1002/mds. 26117

34. Yoon JH, Park DK, Yong SW, Hong JM. Vitamin D deficiency and its relationship with endothelial dysfunction in patients with early Parkinson's disease. J Neural Transm (Vienna) (2015) 122:1685-91. doi: 10.1007/s00702-015-1452-y

35. Topal K, Paker N, Bugdayci D, Ozer F, Tekdos D. Bone mineral density and vitamin D status with idiopathic Parkinson's disease. Osteoporos Int. (2010) 21:S141-S2. doi: 10.1007/s00198-010-1247-9

36. Evatt M, DeLong M, Kumari M, Auinger P, McDermott M, Tangpricha V. High prevalence of hypovitaminosis D status in patients with early Parkinson disease. Arch Neurol. (2011) 68:314-9. doi: 10.1001/archneurol.2011.30

37. Petersen MS, Bech S, Christiansen DH, Schmedes AV, Halling J. The role of vitamin D levels and vitamin D receptor polymorphism on Parkinson's disease in the Faroe Islands. Neurosci Lett. (2014) 561:74-9. doi: 10.1016/j.neulet.2013.12.053

38. Mrabet S, Ben Ali N, Achouri A, Dabbeche R, Najjar T, Haouet S, et al. Gastrointestinal dysfunction and neuropathologic correlations in Parkinson disease. J Clin Gastroenterol. (2016) 50:e85-90. doi: $10.1097 / \mathrm{mcg} .0000000000000606$

39. Serrano-Urrea R, Garcia-Meseguer MJ. Malnutrition in an elderly population without cognitive impairment living in nursing homes in Spain: study of prevalence using the Mini Nutritional Assessment test. Gerontology (2013) 59:490-8. doi: 10.1159/000351763

40. Zhen D, Liu L, Guan C, Zhao N, Tang X. High prevalence of vitamin D deficiency among middle-aged and elderly individuals in northwestern China: its relationship to osteoporosis and lifestyle factors. Bone (2015) 71:1-6. doi: 10.1016/j.bone.2014.09.024

41. Galesanu C, Mocanu V. Vitamin D deficiency and the clinical consequences Rev Med Chir Soc Med Nat Iasi (2015) 119:310-8.

42. Vinh Quoc Luong K, Thi Hoang Nguyen L. Vitamin D and Parkinson's disease. J Neurosci Res. (2012) 90:2227-36. doi: 10.1002/jnr.23115

43. Eyles DW, Smith S, Kinobe R, Hewison M, McGrath JJ. Distribution of the vitamin D receptor and 1 alpha-hydroxylase in human brain. J Chem Neuroanat. (2005) 29:21-30. doi: 10.1016/j.jchemneu.2004. 08.006

44. Peterson AL. A review of vitamin D and Parkinson's disease. Maturitas (2014) 78:40-4. doi: 10.1016/j.maturitas.2014.02.012

45. Tanaka K, Miyake Y, Fukushima W, Kiyohara C, Sasaki S, Tsuboi Y, et al. Vitamin D receptor gene polymorphisms, smoking, and risk of sporadic Parkinson's disease in Japan. Neurosci Lett. (2017) 643:97-102. doi: 10.1016/j.neulet.2017.02.037

46. Gatto NM, Sinsheimer JS, Cockburn M, Escobedo LA, Bordelon Y, Ritz B. Vitamin D receptor gene polymorphisms and Parkinson's disease in a population with high ultraviolet radiation exposure. J Neurol Sci. (2015) 352:88-93. doi: 10.1016/j.jns.2015.03.043

47. Airavaara M, Voutilainen MH, Wang Y, Hoffer B. Neurorestoration. Parkinsonism Relat Disord. (2012) 18(Suppl. 1):S143-6. doi: 10.1016/s1353-8020(11)70045-1 
48. Rcom-H'cheo-Gauthier AN, Meedeniya AC, Pountney DL. Calcipotriol inhibits alpha-synuclein aggregation in SH-SY5Y neuroblastoma cells by a Calbindin-D28k-dependent mechanism. J Neurochem. (2017) 141:263-74. doi: 10.1111/jnc.13971

49. Shah I, Akhtar MK, Hisaindee S, Rauf MA, Sadig M, Ashraf SS. Clinical Diagnostic tools for vitamin D assessment. J Steroid Biochem Mol Biol. (2017) 180:105-117. doi: 10.1016/j.jsbmb.2017.10.003

50. Suzuki M, Yoshioka M, Hashimoto M, Murakami M, Noya M, Takahashi $\mathrm{D}$, et al. Randomized, double-blind, placebo-controlled trial of vitamin D supplementation in Parkinson disease. Am J Clin Nutr. (2013) 97:1004-13. doi: 10.3945/ajcn.112.051664

51. Miyake Y, Tanaka K, Fukushima W, Sasaki S, Kiyohara C, Tsuboi Y, et al. Lack of association of dairy food, calcium, and vitamin D intake with the risk of Parkinson's disease: a case-control study in Japan. Parkinsonism Relat Disord. (2011) 17:112-6. doi: 10.1016/j.parkreldis.2010.11.018
52. Chen H, Zhang SM, Hernan MA, Willett WC, Ascherio A. Diet and Parkinson's disease: a potential role of dairy products in men. Ann Neurol. (2002) 52:793-801. doi: 10.1002/ana.10381

Conflict of Interest Statement: The authors declare that the research was conducted in the absence of any commercial or financial relationships that could be construed as a potential conflict of interest.

Copyright (c) 2018 Luo, Ou, Dutta, Tian, Xiong and Shang. This is an open-access article distributed under the terms of the Creative Commons Attribution License (CC $B Y)$. The use, distribution or reproduction in other forums is permitted, provided the original author(s) and the copyright owner(s) are credited and that the original publication in this journal is cited, in accordance with accepted academic practice. No use, distribution or reproduction is permitted which does not comply with these terms. 